\title{
Update on electron-cloud power deposition for the Large Hadron Collider arc dipoles
}

\author{
Miguel A. Furman* \\ Center for Beam Physics, Lawrence Berkeley National Laboratory, Building 71R0259, \\ 1 Cyclotron Road, Berkeley, California 94720-8211, USA \\ Vernon H. Chaplin ${ }^{\dagger}$ \\ Swarthmore College, 500 College Avenue, Swarthmore, Pennsylvania 19081, USA
}

(Received 30 January 2006; published 20 March 2006)

\begin{abstract}
We revisit the estimation of the power deposited by the electron cloud (EC) in the arc dipoles of the Large Haydron Collider, by means of simulations. We adopt, as simulation input, a set of electron-related parameters closely resembling those used in recent simulations at CERN [F. Zimmermann, in LTC Meeting No. 40, CERN, 2005]. We explore values for the bunch population $N_{b}$ in the range $0.4 \times 10^{11} \leq$ $N_{b} \leq 1.6 \times 10^{11}$, peak secondary electron yield $\delta_{\max }$ in the range $1.0 \leq \delta_{\max } \leq 2.0$, and bunch spacing $t_{b}$ either 25 or $75 \mathrm{~ns}$. For $t_{b}=25 \mathrm{~ns}$ we find that the EC average power deposition per unit length of beam pipe, $d \bar{P} / d z$, will exceed the available cooling capacity, which we take to be $1.7 \mathrm{~W} / \mathrm{m}$ at nominal $N_{b}[\mathrm{~F}$. Zimmermann, in LHC MAC Meeting No. 17, 2005], if $\delta_{\max }$ exceeds $\sim 1.3$, but $d \bar{P} / d z$ will be comfortably within the cooling capacity if $\delta_{\max } \leq 1.2$. For $t_{b}=75 \mathrm{~ns} d \bar{P} / d z$ exceeds the cooling capacity only when $\delta_{\max }>2$ and $N_{b}>1.5 \times 10^{11}$ taken in combination. The rediffused component of the secondary electron emission spectrum plays a significant role: if we artificially suppress this component while keeping $\delta_{\max }$ fixed, $d \bar{P} / d z$ is roughly cut in half for most values of $N_{b}$ explored here, and in this case we find good agreement with earlier results [F. Zimmermann, in LTC Meeting No. 40, CERN, 2005], as expected. We provide a fairly detailed explanation of the mechanism responsible for such a relatively large effect. We assess the sensitivity of our results to numerical simulation parameters, and to physical parameters such as the photoelectric yield, bunch train length, etc. Owing to the lack of detailed knowledge of the electron emission spectrum, the sensitivity of $d \bar{P} / d z$ to the rediffused component appears to be the most significant source of uncertainty in our results. Nevertheless, taking our results as a whole, the condition $\delta_{\max } \leq 1.2$ seems to be a conservative requirement for the cooling capacity not to be exceeded.
\end{abstract}

DOI: 10.1103/PhysRevSTAB.9.034403

PACS numbers: 29.27.Bd

\section{INTRODUCTION}

The discovery of a beam instability induced by the electron cloud (EC) at the Photon Factory (PF) at KEK $[1,2]$ triggered intense experimental and theoretical research activity aimed at assessing a similar effect at $e^{+} e^{-}$colliders [3-7] and the Large Hadron Collider (LHC), [8,9]. Independently, and almost simultaneously with these instabilities studies, it was pointed out by Gröbner that the EC raises two other concerns in the LHC: (a) a potential pressure instability [10] similar to the one observed at the CERN intersecting storage rings when operated in bunched-beam mode [11], and (b) a potentially large power deposition on the walls of the beam screen by the electrons "rattling around" the vacuum chamber under the action of the beam [12]. Since the discovery at the PF electron-cloud effects (ECEs) and their cures have been intensely researched at various laboratories around the world, and have been the subject of various meetings [13-24] and reviews [25-28]. These ECEs are related to the electron-proton instabilities first observed

\footnotetext{
*Email address: mafurman@lbl.gov

Electronic address: http://mafurman.lbl.gov

${ }^{\dagger}$ Email address: vchapli1@ @ swarthmore.edu
}

and studied at Budker Institute for Nuclear Physics in the mid-1960's [29,30], and at the LANL Proton Storage Ring (PSR) since the mid-1980's [31].

The LHC will be the first proton storage ring ever built in which the circular trajectory of the beam will lead to significant synchrotron radiation emitted as a by-product of the particle motion through the bending magnets. At top beam energy this radiation will generate a substantial number of photoelectrons upon striking the chamber. The main uncertainty in the determination of the EC power deposition, however, arises not from the photoelectrons but rather from the compounding effect of secondary electron emission which, when combined with the time structure of the beam, leads to a large amplification factor (typically $2-$ 3 orders of magnitude relative to the photoelectric component) of the average EC density, and strong time fluctuations in the instantaneous power deposition [32,33].

Given that the cryogenic system required for the superconducting magnets was designed before the discovery of the electron-cloud effect, the specification of the cooling capacity of the system did not take into account the contribution to the power deposition by the EC. Since insufficient cooling capacity might require the LHC to operate below the nominally specified beam energy and/or intensity, significant experimental and theoretical effort has 
been devoted at CERN and elsewhere since 1997 to better estimate $d \bar{P} / d z$, to identify the conditions under which the cooling capacity may be exceeded, and to devise mitigation mechanisms if necessary [34]. Early results, based on analytical models and simulations, yielded estimates for $d \bar{P} / d z$ in the range $\sim 0.2$ to $\sim$ several $\mathrm{W} / \mathrm{m}[12,35-38]$, which should be compared to the cooling capacity available for the ECE, $\sim 1.7 \mathrm{~W} / \mathrm{m}$ at nominal bunch intensity [39]. Further work showed a rather strong sensitivity of $d \bar{P} / d z$ to certain parameters pertaining to the secondary electron yield (SEY) and the secondary electron spectrum [32,40-44]. Since some of these parameters are not very well known for the surface material of the LHC vacuum chamber, this sensitivity remains a source of uncertainty in the estimate of the power deposition.

In this article we revisit, in greater detail, our previous estimates of $d \bar{P} / d z[32,36,40,42,43]$ in an arc dipole of the LHC (dipole type "MB" [45]). Although a similarly detailed analysis remains to be carried out for other magnets such as quadrupoles, and for field-free regions, the priority falls on the MB-type dipoles as they account for $\sim 66 \%$ of the circumference and $\sim 70 \%$ of the length of the cryostat. We use here a set of parameters that is close, but not identical, to a set used in Ref. [46]. Some of these parameters are taken from measurements at CERN and elsewhere [47-58]. Although certain parameters still remain to be pinned down, the above measurements have effectively constrained the model for photoemission and secondary emission. The main purpose of this article is to take advantage of these new constraints to provide more detailed, and presumably more realistic, estimates for $d \bar{P} / d z$, and to shift the focus to other parameters that remain to be accurately measured. In addition, we confirm and explain in detail the strong effect that the rediffused electrons have on the average EC charge line density $\bar{\lambda}_{e}$ and on $d \bar{P} / d z$ [44]. When we neglect the rediffused electrons while keeping $\delta_{\max }$ fixed we find good agreement with Ref. [46]—as expected, since the model used in this latter work neglects rediffused electrons. We briefly discuss remaining uncertainties, and the implications of our results for the conditioning process during LHC commissioning. ${ }^{1}$

\section{SIMULATION DETAILS}

\section{A. Beam and chamber}

We simulate the EC buildup when a bunch train is injected into an empty chamber in an arc dipole magnet of length $L=14.2 \mathrm{~m}$ and magnetic field $B=8.39 \mathrm{~T}$. We let the bunch intensity $N_{b}$ range in $(0.4-1.6) \times 10^{11}$. The bunch spacing $t_{b}$ is either 25 or $75 \mathrm{~ns}$, corresponding to 10 or $30 \mathrm{rf}$ buckets, respectively. For $t_{b}=25(75) \mathrm{ns}$ the bunch

\footnotetext{
${ }^{1}$ The overbar notation for $d \bar{P} / d z$ and $\bar{\lambda}_{e}$ indicates an average of the corresponding instantaneous quantities over a time interval $\Delta t$ equal to one batch, $\Delta t=2 \mu \mathrm{s}$ (or longer if explicitly stated).
}

train, or "batch," consists of 72(24) bunches, followed by a gap, for a total length of 810 buckets, or $\sim 2 \mu$ s of beam time. ${ }^{2}$ We represent the cross section of the beam screen by an ellipse of semiaxes $(a, b)=(2.2,1.8) \mathrm{cm}$. For our purposes, this shape is a reasonable approximation to the actual shape, namely, a circle of radius $a$ with flattened top and bottom of full height $2 b$ [59]. A comparison of the EC buildup between the actual shape and the elliptical shape shows that, in many cases, the results are similar [60].

\section{B. Photoelectrons}

In this note we consider only the two most important sources of electrons within the LHC arc chamber, namely, photoemission from the synchrotron radiation striking the walls of the chamber, and secondary electron emission. At top beam energy $\left(E_{b}=7 \mathrm{TeV}\right)$ these two sources dominate over others, such as ionization of residual gas.

At this energy, corresponding to a relativistic factor $\gamma_{b}=7.46 \times 10^{3}$, the beam will emit synchrotron radiation with a critical energy $E_{\text {crit }}=44.1 \mathrm{eV}$ at the rate of $n_{\gamma}^{\prime}=$ $1.27 \times 10^{-2}$ photons per proton per meter of trajectory in the bending magnets of the arcs. ${ }^{3}$ At nominal bunch intensity, $N_{b}=1.15 \times 10^{11}$, this implies $1.45 \times 10^{9}$ radiated photons per bunch per meter. The effective quantum efficiency per penetrated photon, or photoelectron yield $Y_{\text {eff }}$, can be estimated from the photon spectrum, average angle of incidence, and surface properties of the wall. Our simulations take as input the number of photoelectrons generated per proton per unit length of beam traversal, $n_{e}^{\prime}=Y_{\text {eff }} n_{\gamma}^{\prime}$, rather than $n_{\gamma}^{\prime}$ and $Y_{\text {eff }}$ separately, hence we only list $n_{e}^{\prime}$, in units of electrons per proton per meter, or $(e / p) / m$, in Tables I and II; the corresponding range explored for $Y_{\text {eff }}$ is $\sim 0.04-0.1$.

We assume the photoelectrons to be emitted from the walls with a spectrum

$$
\frac{d N}{d E d \Omega} \propto e^{-\left(E-E_{\gamma}\right)^{2} / 2 \sigma_{E \gamma}^{2}} \times \cos ^{2} \theta,
$$

where $E$ is the kinetic energy of the emitted photoelectron, $\theta$ is the emission angle relative to the normal to the surface at the emission point, and the phenomenological parameters $E_{\gamma}$ and $\sigma_{E \gamma}$ are listed in Table I. The overwhelming number of synchrotron photons are radiated in a fan of vertical rms opening angle $\sim \gamma_{b}^{-1}=134 \mu \mathrm{rad}$, and strike the outboard side of the beam screen some $\sim 10 \mathrm{~m}$ downstream of the radiation point, leading to an illuminated strip of rms height $\sigma_{\gamma}=1.4 \mathrm{~mm}$. We assume, following

\footnotetext{
${ }^{2}$ Owing to a misunderstanding we chose a batch length of 810 buckets, while the actual specification is 800 . The extra 10 buckets in the gap have a negligible effect on our results.

${ }^{3}$ This value for $n_{\gamma}^{\prime}$ takes into account only those photons whose energy $h \nu$ is $\geq 4 \mathrm{eV}$, since lower energy photons will not photoemit. Roughly $50 \%$ of the incoherently emitted photons have $h \nu \geq 4 \mathrm{eV}$ [32].
} 
TABLE I. Assumed parameters for EC simulations.

\begin{tabular}{|c|c|c|}
\hline Parameter & Symbol [unit] & Value \\
\hline \multicolumn{3}{|l|}{ Ring and beam parameters } \\
\hline Beam energy & $E_{b}[\mathrm{TeV}]$ & 7 \\
\hline Relativistic beam factor & $\gamma_{b}$ & 7460.5 \\
\hline Beam screen cross section & $\ldots$ & elliptical $^{\mathrm{a}}$ \\
\hline Beam screen semiaxes & $(a, b)[\mathrm{cm}]$ & $(2.2,1.8)^{\mathrm{a}}$ \\
\hline Circumference & $C[\mathrm{~m}]$ & 26658.883 \\
\hline Harmonic number & $h$ & 35640 \\
\hline rf wavelength & $\lambda_{\mathrm{rf}}[\mathrm{m}]$ & 0.748 \\
\hline rf period & $T_{\mathrm{rf}}[\mathrm{ns}]$ & 2.5 \\
\hline Bunch spacing & $t_{b}[\mathrm{~ns}]$ & $25(75)$ \\
\hline Bunch spacing ${ }^{\mathrm{b}}$ & $s_{b}[\mathrm{~m}]$ & $7.48(22.44)$ \\
\hline Bunch spacing ${ }^{\mathrm{b}}$ & ... [buckets] & $10(30)$ \\
\hline Bunch train length ${ }^{c}$ & .. [buckets] & 810 \\
\hline No. bunches per batch ${ }^{\mathrm{b}}$ & $\ldots$ & $72(24)$ \\
\hline Bunch population & $N_{b}$ & $(0.4-1.6) \times 10^{11}$ \\
\hline rms bunch length & $\sigma_{z}[\mathrm{~cm}]$ & 7.5 \\
\hline rms bunch length & $\sigma_{t}[\mathrm{~ns}]$ & 0.25 \\
\hline Longit. bunch profile & $\ldots$ & Gaussian \\
\hline Transverse bunch profile & $\ldots$ & Gaussian \\
\hline Transverse rms bunch sizes & $\left(\sigma_{x}, \sigma_{y}\right)[\mathrm{mm}]$ & $(0.3,0.3)$ \\
\hline Simulated section & $\ldots$ & arc dipole magnet \\
\hline Length of simulated region & $L[\mathrm{~m}]$ & 14.2 \\
\hline Dipole magnet field & $B[\mathrm{~T}]$ & 7.39 \\
\hline \multicolumn{3}{|l|}{ Electron parameters } \\
\hline Peak SEY ${ }^{d}$ & $\delta_{\max }$ & $1.0-2.0$ \\
\hline Photoelectron generation rate ${ }^{\mathrm{d}}$ & $n_{e}^{\prime}[(e / p) / \mathrm{m}]$ & $(0.5-1.2) \times 10^{-3}$ \\
\hline Energy at peak $\mathrm{SEY}^{\mathrm{d}}$ & $E_{\max }[\mathrm{eV}]$ & $218-244$ \\
\hline SEY at 0 energy $^{\mathrm{d}}$ & $\delta(0)$ & $0.31-0.63$ \\
\hline Relative backscattered component at $E_{\max }$ & $\tilde{R}_{e}\left(E_{\max }\right)$ & 0.1 \\
\hline Effective photon reflectivity & $R_{\gamma}$ & 0.2 \\
\hline rms height of illuminated region & $\sigma_{\gamma}[\mathrm{mm}]$ & 1.4 \\
\hline Photoelectron spectrum parameters & $\left(E_{\gamma}, \sigma_{E \gamma}\right)[\mathrm{eV}]$ & $(5,5)$ \\
\hline \multicolumn{3}{|l|}{ Simulation parameters } \\
\hline No. kicks per bunch & $N_{k}$ & 21 \\
\hline (Full bunch length)/(rms bunch length) & $L_{b} / \sigma_{z}$ & 5 \\
\hline Time step & $\Delta t[\mathrm{~s}]$ & $6.25 \times 10^{-11}$ \\
\hline No. primary macroelectrons per bunch passage & $M_{e}$ & 1000 \\
\hline Macroelectron charge at $N_{b}=1 \times 10^{11}$ & $Q[\mathrm{e}]$ & $1.03 \times 10^{6}$ \\
\hline Space-charge grid & $\left(h_{x}, h_{y}\right)[\mathrm{mm}]$ & $6 \times 6$ \\
\hline
\end{tabular}

${ }^{\text {a The elliptical shape used in our simulations is meant as an approximation to the actual shape }}$ (circle of radius $a$ with flattened top and bottom of full height $2 b[59,60]$ ).

${ }^{\mathrm{b}}$ First (second) value is for $t_{b}=25(75) \mathrm{ns}$.

${ }^{\mathrm{c}}$ See footnote 2 .

${ }^{\mathrm{d}}$ See Table II for further details.

Ref. [46], that the effective photon reflectivity is $R_{\gamma}=0.2$ which means that $80 \%$ of the photoelectrons are generated at the illuminated strip with a distribution $d N / d y \propto$ $\exp \left(-y^{2} / 2 \sigma_{\gamma}^{2}\right)$ where $y$ is the vertical direction along the wall relative to the midplane. The remaining $20 \%$ of the photoelectrons are generated uniformly around the perimeter of the cross section of the beam screen (a nonuniform alternative is discussed in Sec. VA 1).

\section{Secondary electrons}

A conditioning process leads to a gradual decrease of the peak value $\delta_{\max }$ of the secondary emission yield (SEY) function $\delta\left(E_{0}\right)$ as the surface is bombarded with electrons or photons, where $E_{0}$ is the electron-wall impact energy. The conditioning process also causes a gradual decrease of the energy $E_{0}=E_{\max }$ at which $\delta\left(E_{0}\right)$ reaches a maximum, 
TABLE II. Other assumed parameters.

\begin{tabular}{lccccc}
\hline \hline$\delta_{\max }$ & $\begin{array}{c}E_{t s, \max } \\
{[\mathrm{eV}]}\end{array}$ & $\begin{array}{c}E_{\max } \\
{[\mathrm{eV}]}\end{array}$ & $\begin{array}{c}\delta(0) \\
\cdots\end{array}$ & $\begin{array}{c}n_{e}^{\prime} \\
{[(e / p) / \mathrm{m}]}\end{array}$ & $\begin{array}{c}R\left(E_{\max }\right) \\
\cdots\end{array}$ \\
\hline 1.0 & 227.6 & 218.2 & 0.31 & $5.08 \times 10^{-4}$ & 0.101 \\
1.1 & 230.0 & 220.8 & 0.35 & $5.80 \times 10^{-4}$ & 0.111 \\
1.2 & 232.4 & 223.4 & 0.38 & $6.53 \times 10^{-4}$ & 0.121 \\
1.3 & 234.7 & 226.0 & 0.41 & $7.25 \times 10^{-4}$ & 0.131 \\
1.5 & 239.5 & 231.1 & 0.47 & $8.71 \times 10^{-4}$ & 0.151 \\
1.7 & 244.2 & 236.3 & 0.53 & $1.02 \times 10^{-3}$ & 0.170 \\
2.0 & 251.4 & 244.0 & 0.63 & $1.23 \times 10^{-3}$ & 0.199 \\
\hline \hline
\end{tabular}

and of $Y_{\text {eff }}$ (and, hence, of $n_{e}^{\prime}$ ). Since we do not precisely know the actual value of $\delta_{\max }$ at the start of the LHC commissioning nor how fast it will decrease during operation, we consider here the range $1 \leq \delta_{\max } \leq 2$, which will almost certainly encompass the values of practical interest. The shifts in $\delta_{\max }, E_{\max }$ and $Y_{\text {eff }}$ are correlated: roughly speaking, they decrease exponentially with the photon or electron dose from the "as-received" initial state to a fully conditioned state. Consequently, following Ref. [46], and consistent with observations, we assume here that $n_{e}^{\prime}$ and $E_{\max }$ interpolate linearly with $\delta_{\max }$ between their measured values at $\delta_{\max }=1$ and $\delta_{\max }=2[47-58,61-63]$.

The secondary emission model used in our simulations is based on a Monte Carlo description of the emission process that is described by phenomenological formulas fitted to experimental data for $\delta\left(E_{0}\right)$ and for the emitted energy spectrum $d \delta / d E$, where $E$ is the emitted electron energy $[64,65]$. The spectrum $d \delta / d E$ exhibits three fairly distinct main components, as illustrated in Fig. 1: elastically reflected electrons $\left(\delta_{e}\right)$, rediffused electrons $\left(\delta_{r}\right)$, and true secondary electrons $\left(\delta_{t s}\right)$, so that $\delta=\delta_{e}+\delta_{r}+\delta_{t s}$.

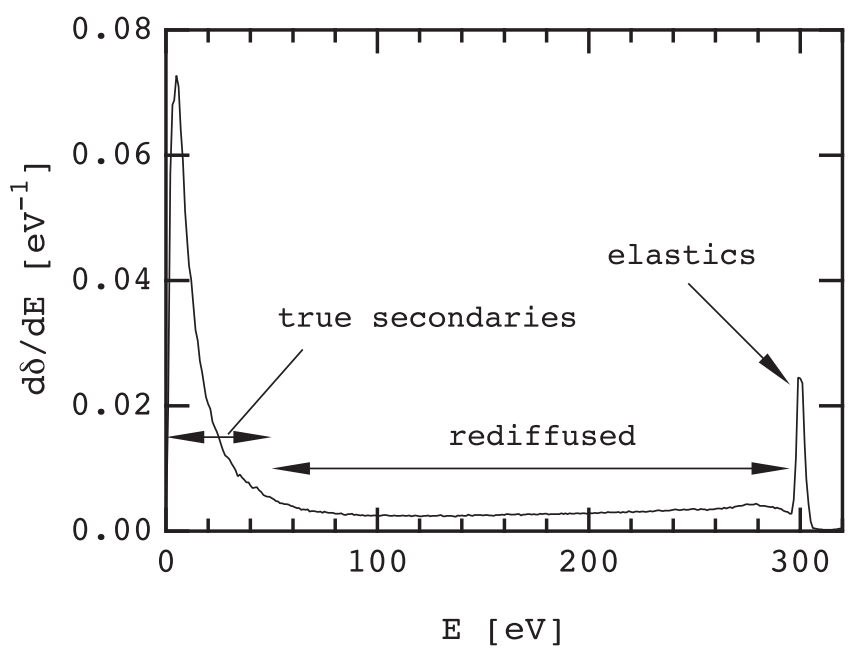

FIG. 1. Sample secondary emission spectrum for $E_{0}=$ $300 \mathrm{eV}$ incident electron energy. For illustrative purposes, the rediffused component is here much larger $\left[\delta_{r}\left(E_{0}\right) \sim 0.75\right.$, or $\sim 37 \%$ of the total] than what we actually used in our simulations ( $\sim 8.5 \%$ ) for comparable values of $E_{0}$.
Each component depends on $E_{0}$, and is determined from the integral

$$
\delta_{i}\left(E_{0}\right)=\int_{E_{1 i}}^{E_{2 i}} d E \frac{d \delta}{d E}, \quad i=e, r, t s,
$$

where the limits $E_{1 i}$ and $E_{2 i}$ are defined by the standard conventions $E_{1 t s}=0, E_{2 t s}=E_{1 r}=50 \mathrm{eV}, E_{2 r}=E_{1 e}=$ $E_{0}-\sigma_{e}, E_{2 e}=E_{0}+\sigma_{e}$ where $\sigma_{e}$ is the rms width of the elastic peak, typically $\sim 5 \mathrm{eV}[66]^{4}{ }^{4}$

We assume that the dependence on incident angle $\theta_{0}$ of each of the three components $\delta_{i}\left(E_{0}\right)$ is taken into account by a multiplicative function $d_{i}\left(\theta_{0}\right)$,

$$
\delta_{i}\left(E_{0}, \theta_{0}\right)=\delta_{i}\left(E_{0}\right) \times d_{i}\left(\theta_{0}\right),
$$

where $\delta_{i}\left(E_{0}\right)$ (with the single argument $E_{0}$ ) represents the yield at normal incidence. The function $d_{i}\left(\theta_{0}\right)$ increases monotonically and smoothly with $\theta_{0}$, with $d_{i}(0)=1$ and $d_{i}(\pi / 2) \simeq 1.5$, where $\theta_{0}=0$ means normal incidence $[64,65]$. The emitted angular distribution is assumed of the form

$$
\frac{d N}{d \Omega} \propto \cos \theta
$$

for any of the three components, where $\theta$ is here the emission angle relative to the normal to the surface at the emission point.

For the purposes of this note we adopt the SEY model corresponding to the copper data in Refs. [64,65], except that here we scale all three components $\delta_{i}\left(E_{0}\right)$ by a common $E_{0}$-independent factor so that $\delta_{\max }$ has the value stated in each simulation case instead of the original value $\delta_{\max }=2.05$. In addition, we assume the above-mentioned linear correlation between $\delta_{\max }, n_{e}^{\prime}$ and $E_{\max }$. The parameter values assumed for each case are listed in Table II. In this table $E_{t s, \text { max }}$ is the value of $E_{0}$ where $\delta_{t s}\left(E_{0}\right)$ reaches its maximum.

We define the absolute and relative backscattered components, respectively, as ${ }^{5}$

$$
\begin{aligned}
& R_{e}\left(E_{0}\right)=\delta_{e}\left(E_{0}\right)+\delta_{r}\left(E_{0}\right), \\
& \tilde{R}_{e}\left(E_{0}\right)=\frac{R_{e}\left(E_{0}\right)}{\delta\left(E_{0}\right)},
\end{aligned}
$$

and are shown in Fig. 2. Since $\delta_{t s}(0)=0$, two measures of the relative magnitude of the backscattered component are $\delta(0)=R_{e}(0)$ and $R_{e}\left(E_{\max }\right)$. Our SEY model has $\tilde{R}_{e}\left(E_{\max }\right)=0.1$ at normal incidence. Had we kept $E_{\max }$ independent of $\delta_{\max }, \tilde{R}_{e}\left(E_{0}\right)$ would also have been independent of $\delta_{\max }$. However, the above-mentioned correlation between $E_{\max }$ and $\delta_{\max }$ introduces a very weak dependence of $\tilde{R}_{e}\left(E_{0}\right)$ on $\delta_{\max }$, as it can be inferred from

\footnotetext{
${ }^{4}$ Obviously, there is no clear separation between the $t s$ and $r$ components when $E_{0} \lesssim 50 \mathrm{eV}$.

"We use the term "backscattered" to jointly describe the rediffused plus elastically reflected electrons.
} 


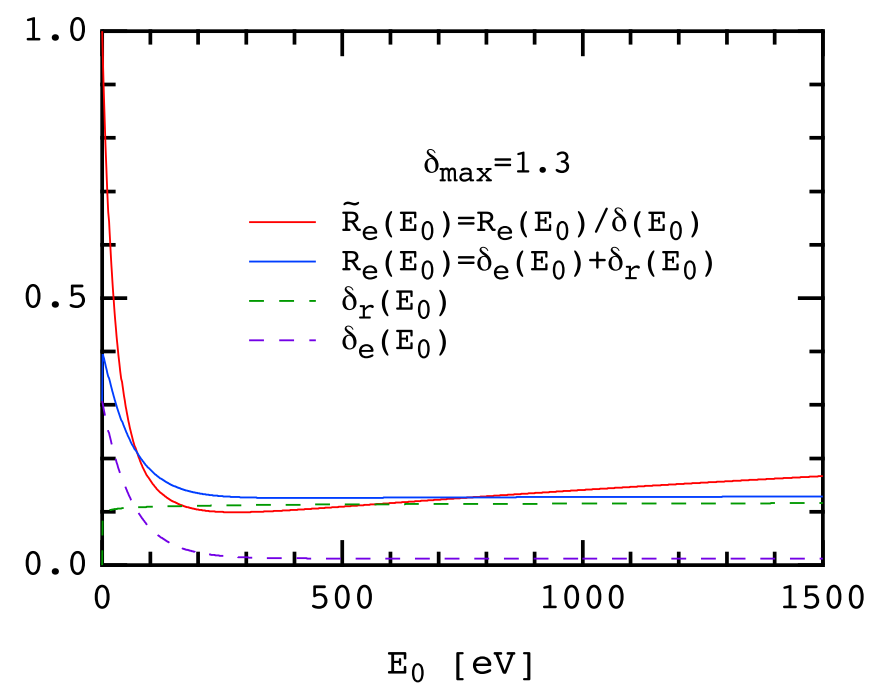

FIG. 2. (Color) The absolute $\left(R_{e}\right)$ and relative $\left(\tilde{R}_{e}\right)$ backscattered components at normal incidence for our model, Eqs. (5), for $\delta_{\max }=1.3$. The relative component has a very weak dependence on $\delta_{\max }$. The elastic and rediffused SEY components $\delta_{e}\left(E_{0}\right)$ and $\delta_{r}\left(E_{0}\right)$ are also shown.

Table II by dividing $R_{e}$ by $\delta_{\max }$. Owing to the mild $E_{0}$ dependence of $R_{e}\left(E_{0}\right), E_{t s, \max }$ is slight larger than $E_{\max }$; see Sec. VA 3 for a discussion.

The scaling of the three components of $\delta$ by a common factor has the consequence that $\delta(0)$ [or, equivalently, $\left.R_{e}(0)\right]$ becomes proportional to $\delta_{\max }$, as seen in Table II. Since we do not know the precise value of $\delta(0)$, this scaling is intended only as a practical step in the parameter exploration, and is not meant to reflect the phenomenology of the secondary emission process. In Sec. IVA we devote special attention to the effect of the rediffused electrons on $d \bar{P} / d z$ by exploring the sensitivity of our results to departures from the proportionality $\delta(0) \propto \delta_{\max }$. For this we carry out simulations in which we fix $\delta_{\max }$ and vary $\delta_{r}$ at the expense of $\delta_{e}$ and $\delta_{t s}$. Further comments are presented in Sec. V.

\section{Simulation technique}

We use here the EC simulation code POSINST, initially developed to study the ECE in the PEP-II positron ring $[6,32,64,65]$. The code has been tested in dedicated experiments at the APS when operated with a positron beam [67], and at the PSR [68]. Good agreement was found between simulations and measurements for the flux of electrons at the walls of the chamber and their energy spectrum. In this code the electrons in the cloud are represented by macroparticles whose number is allowed to change dynamically as the buildup progresses. A number $N_{e}=n_{e}^{\prime} L N_{b}$ of photoelectrons are generated during the passage of one bunch through the dipole magnet. These $N_{e}$ electrons are represented by $M_{e}$ macroelectrons; in most cases presented here we choose $M_{e}=1000$. All macroelectrons have the same charge $Q$, given by $Q / e=N_{e} / M_{e}=n_{e}^{\prime} L N_{b} / M_{e}$. All pri- mary and secondary macroelectrons successively generated during the simulation run have the same charge.

The beam is represented by a prescribed function of space and time which, in the present case, is composed of a succession of proton bunches with tri-Gaussian distribution. The full bunch length, defined to be 5 times the rms length, is divided into $N_{k}-1$ equal-length slices, corresponding to $N_{k}$ kicks. This defines a simulation time step $\Delta t=5 \sigma_{t} /\left(N_{k}-1\right)$, where $\sigma_{t}$ is the rms bunch length in time units. The empty space between bunches is divided into time steps of the same length $\Delta t$. The space-charge (EC self-forces) are computed by means of a $6 \mathrm{~mm} \times$ $6 \mathrm{~mm}$ transverse two-dimensional grid. The self-field is computed and applied to the macroelectrons at every time step.

\section{RESULTS}

Our main results are shown in Fig. 3. For the case $t_{b}=$ $25 \mathrm{~ns}$ and $N_{b}$ close to its nominal value $1.15 \times 10^{11}$ the cooling capacity available for the EC $(1.7 \mathrm{~W} / \mathrm{m})$ is exceeded if $\delta_{\max }$ exceeds $\sim 1.3$. If, however, $\delta_{\max } \leq 1.2$, the power deposition is comfortably below the cooling capacity. Indeed, if we fix $N_{b}=1 \times 10^{11}, d \bar{P} / d z$ shows a clear threshold behavior as a function of $\delta_{\max }$ near $\delta_{\max }=$ 1.2, as seen in Fig. 3(c). For $t_{b}=75$ ns [Fig. 3(b)] the power deposition is well below the cooling capacity unless $\delta_{\max }>2$ and $N_{b}>1.5 \times 10^{11}$ taken in combination.

The values of $d \bar{P} / d z$ in Fig. 3 are obtained from simulations in which a single batch is injected into an empty arc dipole magnet chamber. As discussed in Sec. III B, this single-batch calculation underestimates $d \bar{P} / d z$.

\section{A. Numerical convergence}

In order to assess the numerical convergence of our calculation, we carried out two tests in which we: (i) doubled the number of primary macroelectrons per bunch passage $M_{e}$, and (ii) halved the time step $\Delta t$. In case (i) the macroelectron charge $Q$ is automatically halved while the number of macroelectrons in existence at any given time is doubled. In case (ii) the number of kicks per bunch $N_{k}$ is 41 instead of 21. We carried out these two tests one at a time, not in combination, and only for the case defined by $t_{b}=$ $25 \mathrm{~ns}, \delta_{\max }=1.3$, and $N_{b}=1 \times 10^{11}$, which we call the "reference case." Other parameters for this case are listed in Table I, and the 4th line in Table II. As seen in Fig. 4 showing $\lambda_{e}$ vs time, there is good agreement with the reference case. Other quantities such as the electron energy spectrum (not shown), also exhibit good agreement. These results strongly suggest, but do not conclusively prove, adequate numerical convergence.

\section{B. Two and three batches}

In order to test for steady-state conditions, we carried out, for the reference case, a simulation of the EC buildup during two successive batches. As seen in Fig. 5, it is clear 

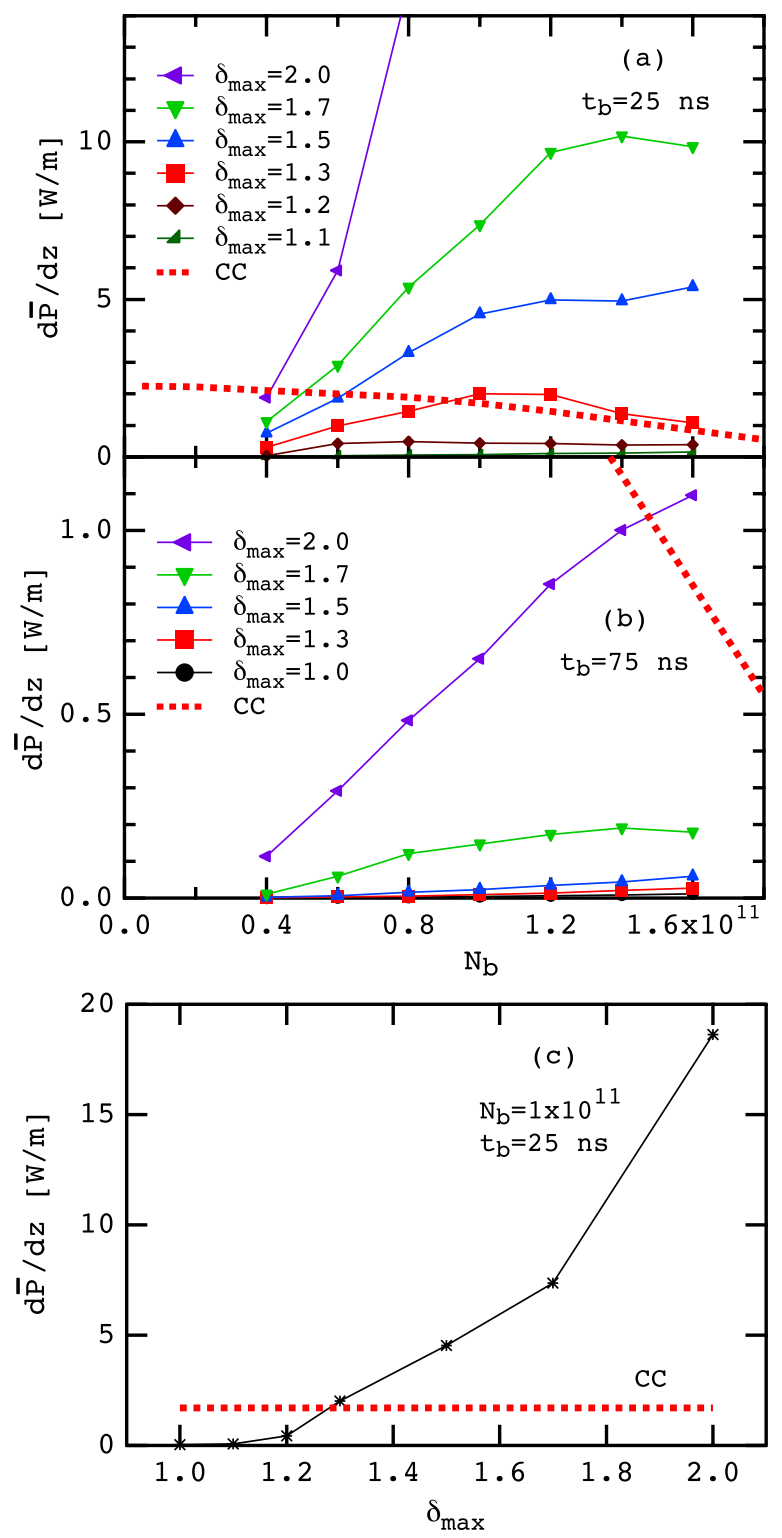

FIG. 3. (Color) Average power deposition. (a) and (b): $d \bar{P} / d z$ vs $N_{b}$ for $t_{b}=25$ and $75 \mathrm{~ns}$, respectively, (c): $d \bar{P} / d z$ vs $\delta_{\max }$ for fixed $N_{b}=1 \times 10^{11}$ and $t_{b}=25 \mathrm{~ns}$. The value of $\delta_{\max }$ labels a specific choice of parameters, corresponding to each line in Table II. CC: cooling capacity of the cryogenic system available for the ECE at high luminosity with $25 \%$ contingency [39]. For $t_{b}=25 \mathrm{~ns}$ and $\delta_{\max }=2, d \bar{P} / d z$ saturates at $\sim 22 \mathrm{~W} / \mathrm{m}$ at $N_{b} \sim$ $1.6 \times 10^{11}$ (off scale). The values of $d \bar{P} / d z$ are obtained from a single batch injected into an empty chamber. The steady-state values are obtained by multiplying these values by $\sim 1.4$. The nominal LHC specification is $N_{b}=1.15 \times 10^{11}$.

that it takes two batches for the EC to sensibly reach steady state. Although $\lambda_{e}(t)$ clearly reaches saturation during the 1 st batch, this saturated value is reached significantly earlier during the $2 \mathrm{nd}$ batch because the remnant of the $\mathrm{EC}$ at the end of the first gap seeds the buildup during the 2nd batch passage. Consequently, the estimate of $d \bar{P} / d z$ during the 2 nd batch $(2<t<4 \mu \mathrm{s})$ is $2.8 \mathrm{~W} / \mathrm{m}$, as opposed to

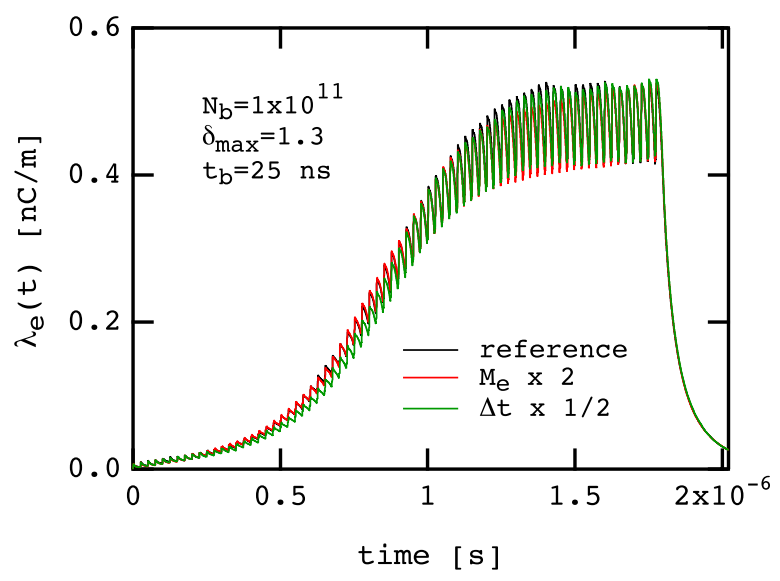

FIG. 4. (Color) $\lambda_{e}(t)$ vs time for: reference case; doubling $M_{e}$; and halving $\Delta t$.

$2 \mathrm{~W} / \mathrm{m}$ during the $1 \mathrm{st}$ batch $(0<t<2 \mu \mathrm{s})$. These results imply that the values shown in Fig. 3 must be scaled up by a factor $\sim 1.4$ to obtain the steady-state estimates of $d \bar{P} / d z$. This issue is discussed in more detail in Sec. VI.

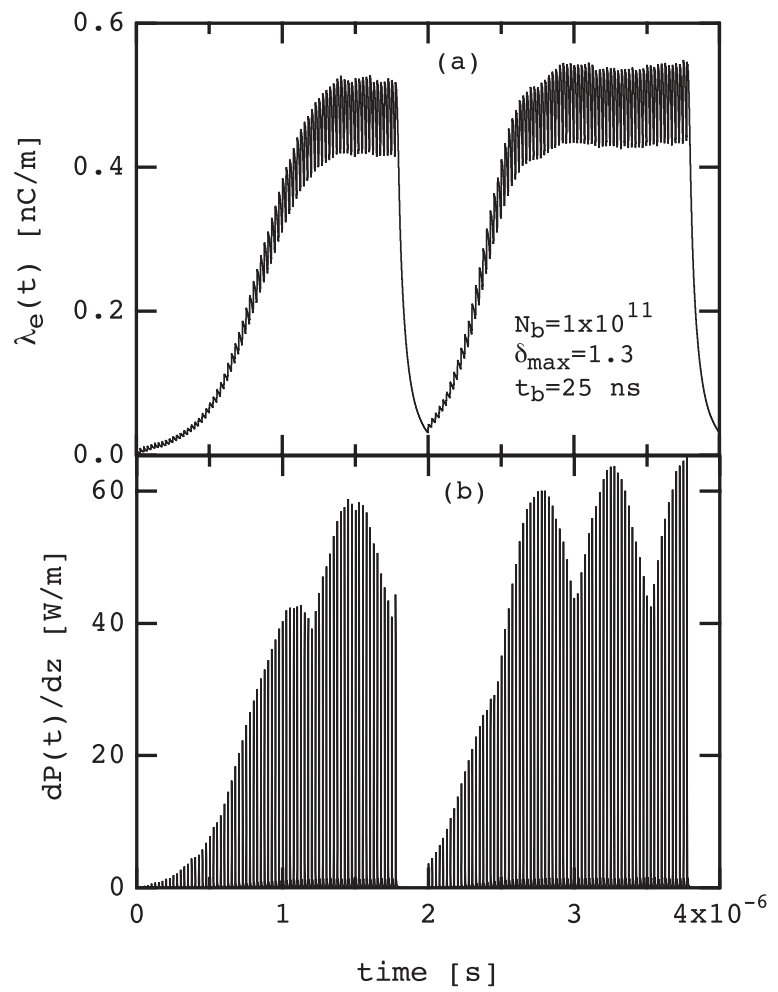

FIG. 5. $\lambda_{e}(t)$ and $d P(t) / d z$ vs time for two batches. $\lambda_{e}(t)$ saturates at $\sim 0.5 \mathrm{nC} / \mathrm{m}$, or $\sim 25 \%$ of the average beam line density, $\quad \bar{\lambda}_{b}=e N_{b} / s_{b}=2.14 \mathrm{nC} / \mathrm{m}$. The power deposition averaged during the $1 \mathrm{st}$ batch is $2 \mathrm{~W} / \mathrm{m}$ (this is the value shown in Fig. 3(a) for $\delta_{\max }=1.3$ and $N_{b}=1 \times 10^{11}$ ), but it is $2.8 \mathrm{~W} / \mathrm{m}$ when averaged during the $2 \mathrm{nd}$ batch. The slow oscillations in $d P(t) / d z$, with a period $\sim 0.5 \mu \mathrm{s}$, are discussed in Sec. VC. 
The fact that the values of $\lambda_{e}(t)$ at the beginning and at the end of the 2nd batch [Fig. 5(a)] are roughly equal strongly suggests that a steady state is sensibly reached after only two batch passages. We confirmed this by running a simulation with three batches (not shown), which showed that the EC rise time and saturation level in the 2nd and 3rd batches are almost identical.

\section{Twice the photoemission rate}

Since the value of $Y_{\text {eff }}$ is not accurately known for the chamber surface under actual operating conditions, we carried out a test case in which the photoelectron generation rate $n_{e}^{\prime}$ was twice the reference value (Table II, 4th line). As seen in Fig. 6, it is clear that although the initial rate of increase of $\lambda_{e}(t)$ is twice the reference case, as it should be expected, the saturated value is essentially unchanged. As a result, the estimated $d \bar{P} / d z$ (not shown) is only $\sim 10 \%$ larger than the reference case. This result suggests that, at least in steady state, $d \bar{P} / d z$ is not very sensitive to other details of photoemission either, such as the geometrical distribution of the photoelectrons.

\section{THE EFFECT OF REDIFFUSED ELECTRONS}

\section{A. Sensitivity to $\boldsymbol{\delta}_{\boldsymbol{r}}$}

In the early simulations for the LHC arc dipoles it was noted that when the backscattered electrons were included in the simulation model, the estimates for $d \bar{P} / d z$ increased significantly compared to the case in which only the true secondary electrons were taken into account $[32,38,41]$. The backscattered electrons modify the SEY function $\delta\left(E_{0}\right)$ only for $E_{0} \lesssim 20 \mathrm{eV}$, chiefly by adding a nonzero contribution to $\delta(0)$. Given that $\delta_{\max }$ was kept fixed in those simulations, it was puzzling at first that a relatively small change in $\delta\left(E_{0}\right)$ would lead to a large effect in the estimate of $d \bar{P} / d z$.

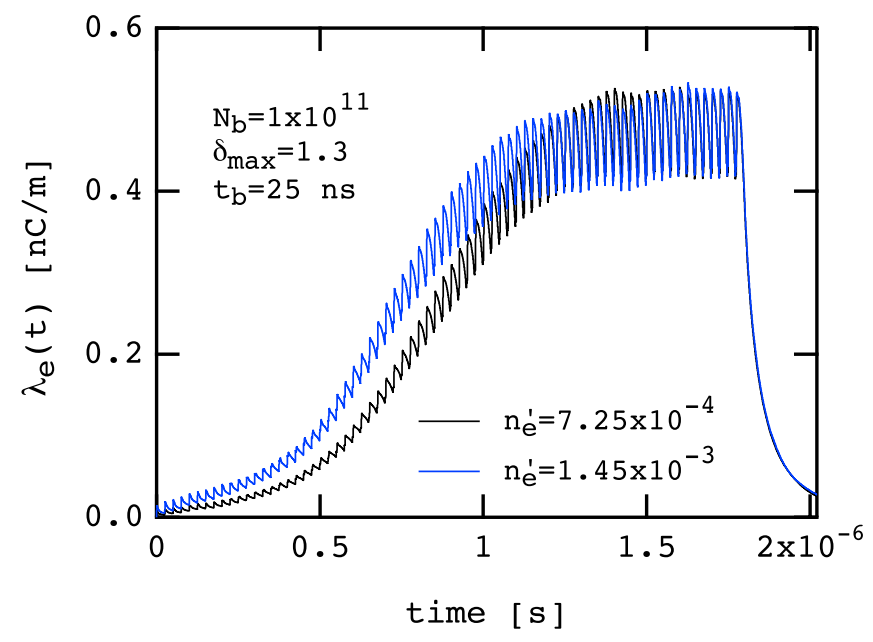

FIG. 6. (Color) $\lambda_{e}(t)$ vs time when the photoemission rate is doubled while all other quantities are held fixed.
Before explaining the mechanism (Sec. IV B), we first illustrate the sensitivity of $d \bar{P} / d z$ to $\delta_{r}$ by considering three cases for the secondary emission spectrum for fixed $\delta_{\max }=1.3$, which we call R ("reference"), NR ("no rediffused") and HR ("high rediffused"). The reference case $\mathrm{R}$ is defined in Sec. III A. In cases NR and HR we adjusted $\delta_{r}$ at the expense of $\delta_{e}$ and $\delta_{t s}$ by scaling these latter two components by a common factor so that $\delta_{\max }$ remained fixed. Figure 7(a) shows the three SEY curves; they differ very little from each other in the energy range of interest, typically below a few hundred eV. Figure 7(b) shows the corresponding simulation results for $\lambda_{e}(t)$ for a single batch.

The main results for $d \bar{P} / d z$ for cases $\mathrm{R}$ and NR are shown in Fig. 8 for $t_{b}=25$ ns. Case R leads to a factor $\sim 2$ larger power deposition than for case NR. The results for this latter case are in good agreement with those in Ref. [46] — as it should be expected, since the model used to obtain these latter results does not include rediffused electrons.
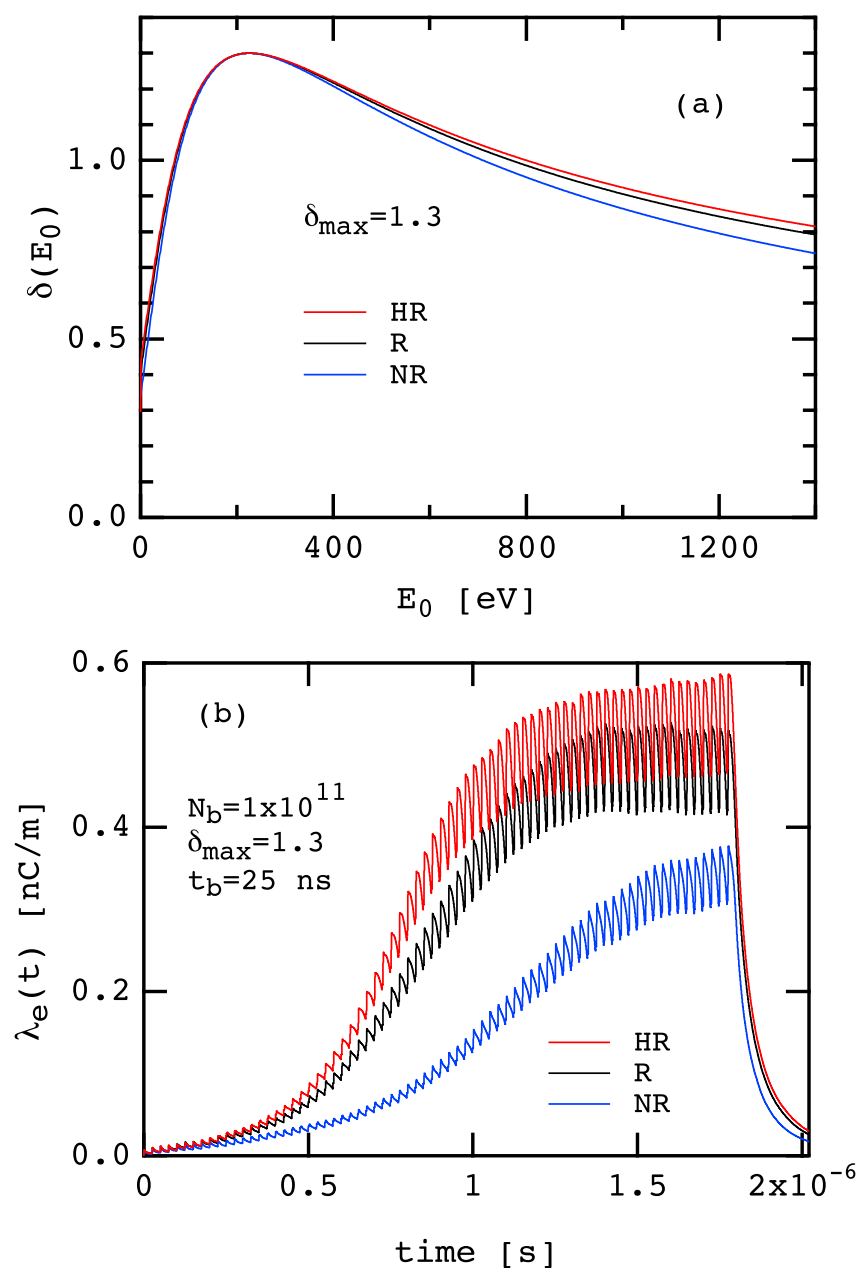

FIG. 7. (Color) (a) The SEY function $\delta\left(E_{0}\right)$ at normal incidence for $\delta_{\max }=1.3$, and (b) the results for $\lambda_{e}(t)$ vs time. R: reference case; NR: no-rediffused case; HR: high-rediffused case. 


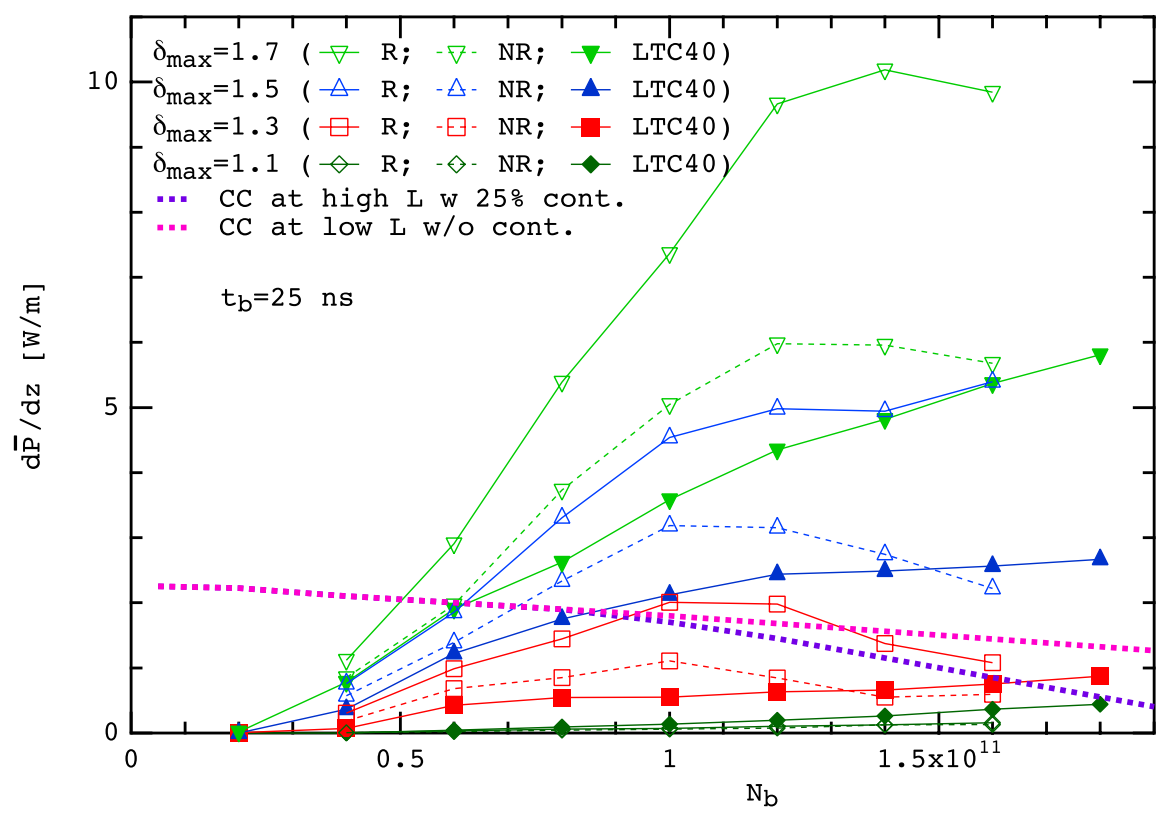

FIG. 8. (Color) $d \bar{P} / d z$ vs $N_{b}$. R: full SEY model. NR: no-rediffused model. LTC40: results from Ref. [46]. CC: cooling capacity available for the ECE at high luminosity with a $25 \%$ contingency, and at low luminosity without contingency [39]. $d \bar{P} / d z$ was obtained from a time average over a single batch. The falloff of $d \bar{P} / d z$ at high $N_{b}$, especially for the NR cases, is probably due to the decrease of $\delta\left(E_{0}\right)$ when the average electron-wall collision energy $\bar{E}_{0}$ exceeds $E_{\max }$.

TABLE III. Three cases for $R_{e}\left(\delta_{\max }=1.3, N_{b}=1 \times 10^{11}, t_{b}=25 \mathrm{~ns}\right)$.

\begin{tabular}{lccccccccc}
\hline \hline Case & $\delta_{e}(0)$ & $\delta_{r}(0)$ & $R_{e}(0)$ & $E_{\max }[\mathrm{eV}]$ & $\delta_{e}\left(E_{\max }\right)$ & $\delta_{r}\left(E_{\max }\right)$ & $R_{e}\left(E_{\max }\right)$ & $\bar{\lambda}_{e}[\mathrm{nC} / \mathrm{m}]$ & $d \bar{P} / d z[\mathrm{~W} / \mathrm{m}]$ \\
\hline $\mathrm{NR}$ & 0.34 & 0.00 & 0.34 & 224.9 & 0.022 & 0.00 & 0.02 & 0.14 & 1.1 \\
$\mathrm{R}$ & 0.31 & 0.09 & 0.41 & 226.0 & 0.020 & 0.11 & 0.13 & 0.25 & 2.0 \\
$\mathrm{HR}$ & 0.30 & 0.13 & 0.43 & 226.5 & 0.019 & 0.16 & 0.18 & 0.28 & 2.4 \\
\hline \hline
\end{tabular}

Table III provides additional details on the input parameters and the corresponding simulation results for this sensitivity exercise. The values quoted here for $\bar{\lambda}_{e}$ and $d \bar{P} / d z$ are obtained from a single-batch simulation for $N_{b}=1 \times$ $10^{11}$ and $t_{b}=25 \mathrm{~ns}$. It is interesting to note that both $\bar{\lambda}_{e}$ and $d \bar{P} / d z$ exhibit an approximately linear dependence on $\delta_{r}(0)$. Defining $d \bar{P} / d z=c_{0}+c_{1} \delta_{r}(0)$, one obtains $c_{0} \simeq$ 1.1 and $c_{1} \simeq 10$, both in $\mathrm{W} / \mathrm{m}$, implying a rather strong sensitivity to $\delta_{r}(0)$. Repeating the same exercise for the case $\delta_{\max }=1.2$ (not shown in Table III) yields $c_{0} \simeq 0.17$ and $c_{1} \simeq 5 \mathrm{~W} / \mathrm{m}$, a significantly weaker dependence. Although the approximately linear dependence is suggestive, one cannot assess from these results its significance nor its range of applicability because $\delta_{r}(0)$ was not varied independently of the other SEY parameters, and because the range explored was rather narrow.

\section{B. Mechanism}

In Ref. [44] we sketched an explanation for the relatively large effect of the rediffused electrons. The explanation focused not on the SEY function $\delta\left(E_{0}\right)$ but on the back- scattered energy spectrum, which is qualitatively different from the true secondary spectrum for most values of most values of $E_{0}$, not just at low energy. In this section we provide a more detailed explanation than that provided in Ref. [44] by contrasting simulation details for cases $R$ and NR.

Figure 10 shows the average electron-wall collision energy for a subset of four bunches in the batch. Case R leads to two waves of electrons striking the walls: the 1 st wave is made up of the electrons in the bulk of the chamber that were kicked by the beam and struck the walls some $3 \mathrm{~ns}$ after the bunch passage. The 2nd wave is mostly composed of rediffused electrons that were generated when the 1 st wave hit the wall. This 2 nd wave is largely absent in case NR. For the same time interval, Fig. 11 shows the effective $\operatorname{SEY}^{6} \delta_{\text {eff }}(t)$ as a function of time $t$. The 1 st wave leads to substantially the same $\delta_{\text {eff }}$ for both cases

\footnotetext{
${ }^{6} \delta_{\text {eff }}(t)$ is defined to be the SEY averaged over all electronwall collision events during the time window $(t, t+\Delta t)$, where $\Delta t=1 \mathrm{~ns}$.
} 


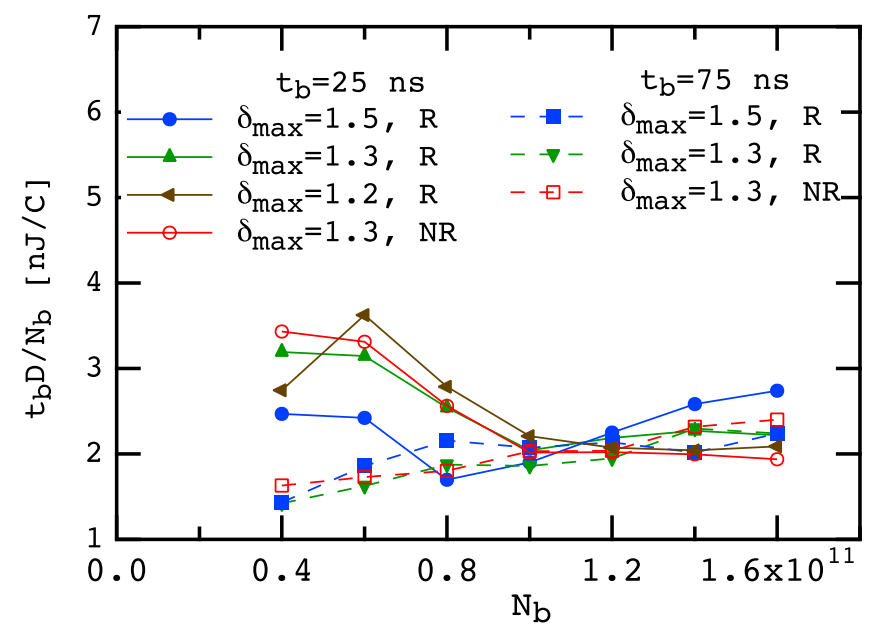

FIG. 9. (Color) The quantity $K \equiv t_{b} D / N_{b}$ vs $N_{b}$ for $t_{b}=25$ and $75 \mathrm{~ns}$, various values of $\delta_{\max }$, and cases R and NR.

$\mathrm{R}$ and NR, but the 2nd wave leads to an enhancement in case $\mathrm{R}$ relative to case NR owing to the higher average electron-wall collision energy $\left(\bar{E}_{0} \sim 100 \mathrm{eV}\right.$, as seen in Fig. 10, where $\delta\left(E_{0}\right)$ rises above unity). The larger $\delta_{\text {eff }}$, in turn, leads to roughly twice $\bar{\lambda}_{e}$ (Fig. 12) for case R relative to case NR, which leads to a higher $d P(t) / d z$ (Fig. 13). The 2nd wave of electrons deposits a small amount of additional energy.

It should be remarked that, just like for case $\mathrm{R}, d \bar{P} / d z$ computed from the 1st batch in case NR also underestimates the steady-state value by $\sim 40 \%$. Indeed, for the 1 st batch in case NR in Fig. 12 we obtain $1 \mathrm{~W} / \mathrm{m}$, while for the

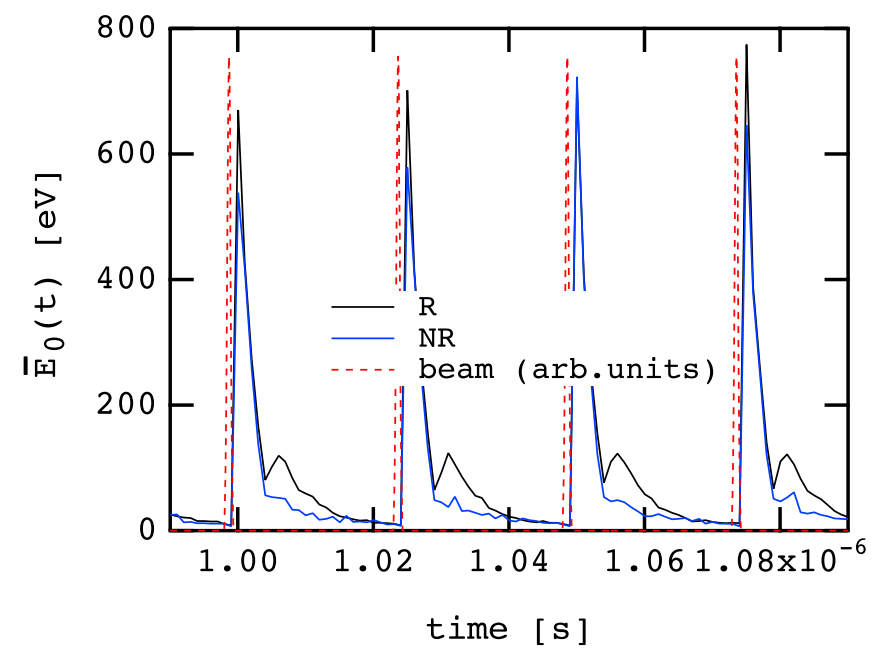

FIG. 10. (Color) Average electron-wall collision energy per electron, $\bar{E}_{0}$, vs time for bunches \#41-\#44 in the 1st batch. R: full model. NR: no-rediffused model. Red dotted line: beam signal (arbitrary units). Some $\sim 3$ ns after the bunch passage the electrons kicked by the beam strike the walls. Some $\sim 5$ ns later, a second wave of electrons hits the walls, most of which are rediffused electrons generated when the 1st wave struck the wall. The 2nd wave is substantially absent in the NR case.

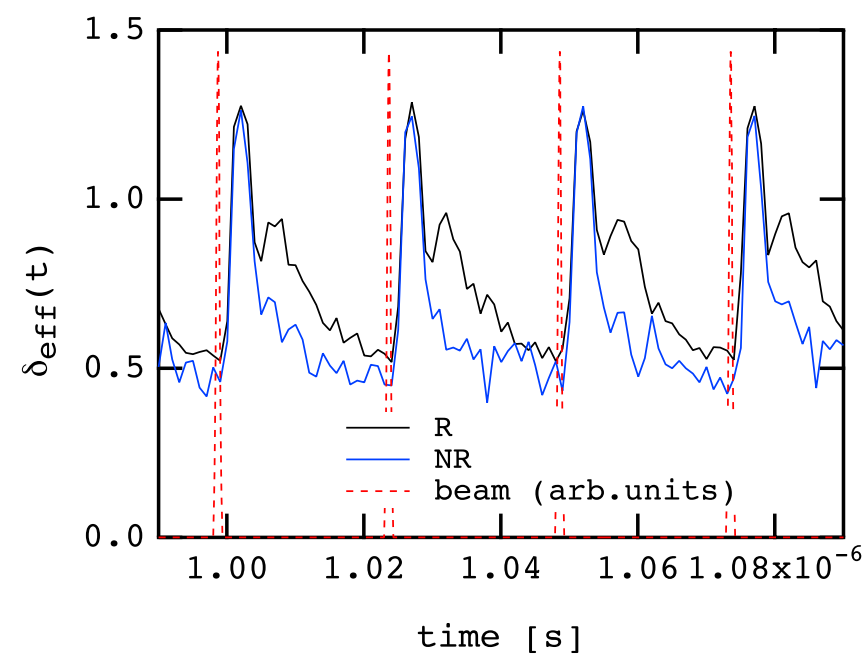

FIG. 11. (Color) $\delta_{\text {eff }}(t)$ vs time for the same bunches shown in Fig. 10. The 2nd wave of electrons leads to a higher effective SEY in case R compared with case NR owing to their higher average wall collision energy.

2nd batch (not shown) we obtain $1.4 \mathrm{~W} / \mathrm{m}$. For case R the corresponding results are $2 \mathrm{~W} / \mathrm{m}$ and $2.8 \mathrm{~W} / \mathrm{m}$, respectively [Fig. 5(b)].

As explained above, most of the energy is deposited by the electrons in the bulk of the chamber upon being kicked by the beam ( 1 st wave of electrons striking the walls). This suggests that $d \bar{P} / d z$ is directly proportional to $\bar{\lambda}_{e}$. This is indeed borne out by our results: for case NR $\bar{\lambda}_{e}$ is 0.14 and $0.22 \mathrm{nC} / \mathrm{m}$ for the $1 \mathrm{st}$ and $2 \mathrm{nd}$ batch, respectively, (not shown), while for case $\mathrm{R}$ the corresponding results are 0.25 and $0.37 \mathrm{nC} / \mathrm{m}$ [Fig. 5(a)]. Defining the ratio

$$
D \equiv \frac{d \bar{P} / d z}{\bar{\lambda}_{e}}
$$

we arrive at the empirical result $D \simeq 8-10 \mathrm{~W} / \mathrm{nC}$ for $N_{b}=$ $1 \times 10^{11}$ and $t_{b}=25 \mathrm{~ns}$. This value of $D$ is found to be the same for either 1 st or 2 nd batch, for any of the three cases $\mathrm{R}, \mathrm{NR}$, and $\mathrm{HR}$, and for $\delta_{\max }=1.2$ or 1.3 .

When $N_{b}$ and $t_{b}$ vary away from the above values, we find that the scaling $D \propto N_{b} / t_{b}$ is qualitatively valid. ${ }^{7}$ Figure 9 shows $K \equiv t_{b} D / N_{b}$ plotted vs $N_{b}$. If the scaling were perfect, $K$ would be a constant independent of $N_{b}$ and all curves would coincide. Given the range of conditions spanned by the data in Fig. 9, we consider the approximate overlap of the curves, especially for $N_{b}>1 \times 10^{11}$, a reasonably good indication of the validity of the scaling. Combining these results yields the approximate empirical

\footnotetext{
${ }^{7}$ A necessary (but not sufficient) condition for the validity of the scaling analysis for $D$ is that the time interval $\Delta t$ used to compute the averages $\bar{\lambda}_{e}$ and $d \bar{P} / d z$ be $\gg t_{b}(\Delta t=2 \mu \mathrm{s}$ in this article).
} 


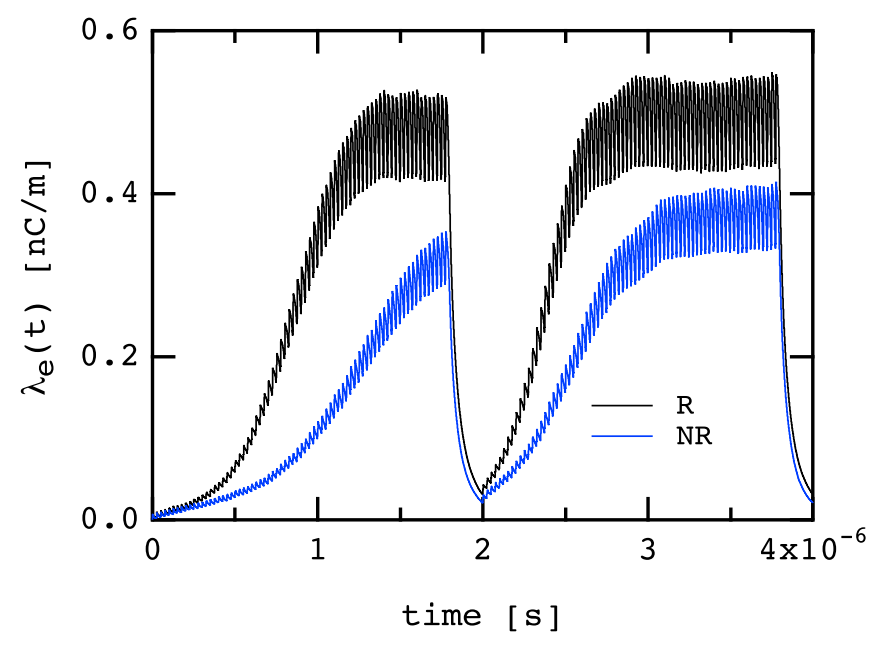

FIG. 12. (Color) $\lambda_{e}(t)$ vs time. The higher $\delta_{\text {eff }}$ in model R leads to $\sim$ twice $\bar{\lambda}_{e}$ in the 1 st batch, as compared with case NR.

formula (see also Sec. V D),

$$
\frac{d \bar{P}}{d z} \simeq K \frac{\bar{\lambda}_{e} N_{b}}{t_{b}}, \quad K \simeq 2 \mathrm{~nJ} / \mathrm{C} .
$$

\section{DISCUSSION}

\section{A. Differences with Ref. [46] \\ 1. Photoelectron distribution}

In our simulations we have assumed that an effective fraction $R_{\gamma}=0.2$ of the photons that strike the outboard side of the beam screen walls are diffusively reflected, and that these are, effectively, evenly redistributed around the perimeter of the cross section of the beam screen. In

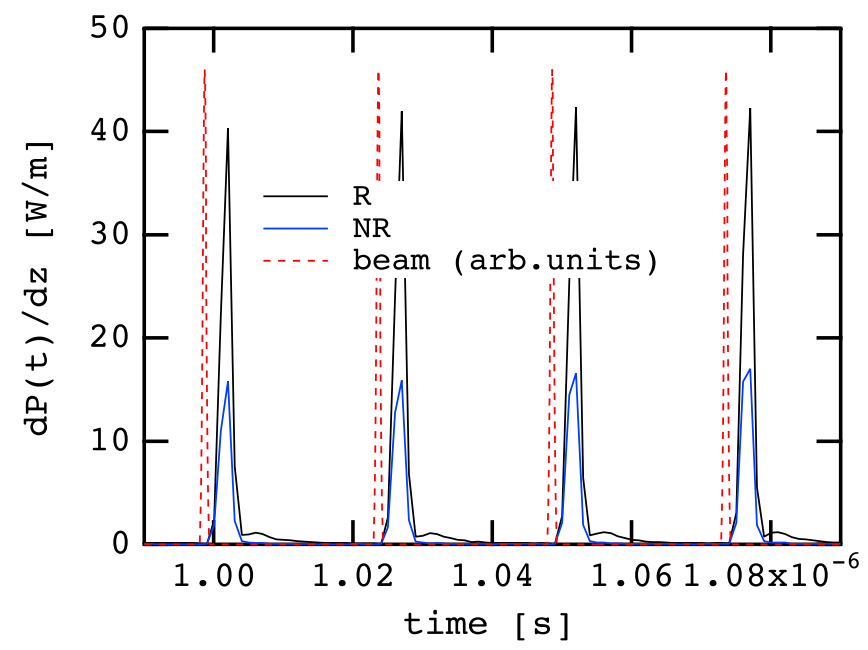

FIG. 13. (Color) $d P(t) / d z$ vs time. The higher values of $\lambda_{e}(t)$ for case $\mathrm{R}$ leads to $\sim$ twice the power deposition relative to the NR case. Most of the power is deposited by the 1st wave of electrons, but in case $\mathrm{R}$ an additional $5-10 \%$ is deposited by the 2 nd wave of electrons.
Ref. [46], on the other hand, photoemission from the diffusively reflected photons was assumed to be enhanced at the point diametrically opposite to the illuminated strip with a weight $\cos ^{n} \phi$, where $\phi$ is the angle relative to the midplane. However, the results for $d \bar{P} / d z$ for $n=2$ or $n=$ 3 showed only a small difference [33]. Other tests confirmed a weak dependence on the details of photoemission [69]. This weak dependence, which is consistent with our results in Sec. III C, should be expected because, for typical LHC parameters, $d \bar{P} / d z$ in steady state is dominated by secondary electrons. The photoelectrons play a role of seeds of the EC buildup, but once secondary emission becomes dominant, the EC distribution in space, time and energy is essentially determined by the SEY, the beam, and the chamber geometry.

Besides the $\cos ^{n} \phi$ factor, the electron emission spectrum in Ref. [46] assumes $E_{\gamma}=7 \mathrm{eV}$ instead of $5 \mathrm{eV}$, as we assume here. For the above reasons, this difference should have a negligible effect on the results. We conclude that the photoelectron distribution does not significantly affect the estimate of $d \bar{P} / d z$, at least not in steady state.

$$
\text { 2. } \delta(0) \propto \delta_{\text {max }}
$$

Some of the parameters in the model of electron emission we have used are correlated, as specified in Table II. The correlations between $\delta_{\max }, E_{\max }$, and $n_{e}^{\prime}$ discussed in Sec. IIC bring the input to our simulations closer to the assumptions used in Ref. [46]. On the other hand, as explained towards the end of Sec. II C, we have, for convenience, introduced the proportionality $\delta(0) \propto \delta_{\max }$ that is not used in Ref. [46], where $\delta(0)$ was kept fixed at $\delta(0)=0.5$. In future calculations we intend to decouple $\delta(0)$ from $\delta_{\max }$; the analysis of the three cases R, NR and HR in Sec. IVA represents our present attempt in this direction.

$$
\text { 3. } E_{\text {max }} \neq E_{t s \text {, } \text { ax }}
$$

The current version of POSINST takes as input the energy $E_{t s, \max }$ at which the true secondary component $\delta_{t s}\left(E_{0}\right)$ of the SEY has a maximum; the value $E_{\max }$ where $\delta\left(E_{0}\right)$ has its peak is an output of the code. Since $\delta\left(E_{0}\right)=\delta_{t s}\left(E_{0}\right)+$ $R_{e}\left(E_{0}\right)$ and $R_{e}\left(E_{0}\right)$ has a weak dependence on $E_{0}, E_{\max }$ is slightly different from $E_{t s, \max }$, as it can be seen in Table II. In order to test the effect of this shift on our results, we ran one case for $\delta_{\max }=1.3$ and $N_{b}=1 \times 10^{11}$ for which $E_{\max }$, rather than $E_{t s, \max }$, had the value $234.7 \mathrm{eV}$ (see Table II). To achieve this, we chose $E_{t s, \max }=242.8 \mathrm{eV}$ instead of $234.7 \mathrm{eV}$. The result was $d \bar{P} / d z \simeq 2.1 \mathrm{~W} / \mathrm{m}$, a $5 \%$ increase over the reference case. We conclude that the slight energy shift in $E_{\max }$ is not significant.

The simulations in Ref. [46] show a decrease of $d \bar{P} / d z$ with increasing $E_{t s \text {,max }}$ in the parameter regime explored. We do not have a definitive explanation for the opposite sign of the effect relative to our results. However, given the numerical accuracy in our calculations and the smallness 
of the absolute value of the effect, we cannot ascertain that the sign of the shift is meaningful in this specific case.

\section{Angular dependence in secondary emission}

In Ref. [46] both the incident-angle dependence of the SEY and the emitted angular distribution of the secondary electrons are treated differently than we do here, described by Eqs. (3) and (4). Specifically, in Ref. [46], the yield $\delta_{e}$ does not depend on $\theta_{0}$, and the elastically backscattered electrons are emitted specularly relative to the incident electron. The true secondary electrons, however, are treated in a qualitatively similar fashion as we do here, and rediffused electrons are not considered.

While a more careful check remains to be carried out, all our simulation results show that the average over all electron-wall collisions during the run yields $\left\langle\cos \theta_{0}\right\rangle>$ 0.7 , with 0.8 being a more typical value. This indicates close-to-normal incidence [typically, $d_{i}\left(\theta_{0}\right) \lesssim 1.1$ ], hence the $\theta_{0}$ dependence of the secondary yield is not a significant factor. As for the angular distribution of the emitted electrons, the difference between the two approaches probably has a small effect on the estimate of $d \bar{P} / d z$ at least in steady state, because in this regime the distribution and intensity of the EC are essentially determined by the strong dipole magnetic field, the SEY, and the beam intensity.

\section{B. Cimino-Collins results for $\boldsymbol{\delta}_{\boldsymbol{e}}\left(\boldsymbol{E}_{\mathbf{0}}\right)$}

A set of delicate measurements of $\delta\left(E_{0}\right)$ and $d \delta / d E$ for copper samples at low temperature $(T \simeq 9 \mathrm{~K})$ carried out at CERN exhibits an upturn in $\delta\left(E_{0}\right)$ as $E_{0}$ decreases below $\sim 20 \mathrm{eV}$, reaching $\delta(0) \simeq 1$ [63]. ${ }^{8}$ The data exhibit the usual conditioning effect whereby $\delta_{\max }$ gradually decreases with electron bombardment. However, the data also exhibit the novel feature that $\delta\left(E_{0}\right)$ is insensitive to electron bombardment for $E_{0} \lesssim 10-20 \mathrm{eV}$. Measurements of the spectrum $d \delta / d E$ for several values of $E_{0}$ allowed the extraction of $\delta_{e}\left(E_{0}\right)$ and $\delta_{r}\left(E_{0}\right)+\delta_{t s}\left(E_{0}\right)$, which showed that $\delta_{e}\left(E_{0}\right) \rightarrow 1$ in the limit $E_{0} \rightarrow 0$ regardless of the state of conditioning of the sample, while $\delta_{r}\left(E_{0}\right)+\delta_{t s}\left(E_{0}\right) \rightarrow 0$ in the same limit. Since $\delta_{t s}\left(E_{0}\right) \rightarrow 0$ in this limit $[64,65]$, these measurements imply $\delta_{r}(0) \simeq 0$, compared with the value $\sim 0.1$ we assume here for the reference case $\mathrm{R}$. On the other hand the measured value $\delta_{e}(0) \simeq 1$ is significantly larger than our assumed value $\left[\delta_{e}(0) \sim 0.3\right]$ for the same case R. ${ }^{9}$

EC simulations showed that $\bar{\lambda}$ and $d \bar{P} / d z$ are significantly higher when a model of the SEY that included the

\footnotetext{
${ }^{8} \mathrm{An}$ indication of this upturn is apparent in another set of measurements: see Ref. [54], Fig. 5.

${ }^{9}$ The extraction of the three separate components $\delta_{e}, \delta_{r}$, and $\delta_{t s}$ from the data, and the simulation study of the consequences of these on the EC seem interesting and desirable, but this falls outside the scope of this article.
}

above-mentioned upturn near $E_{0}=0$ was used as input to the simulations compared with those in which $\delta_{e}\left(E_{0}\right)$ was artificially suppressed [70]. We believe that this result is explained by the mechanism described in Sec. IV B, since this mechanism applies to the elastically backscattered electrons as well as to the rediffused. Confirmation of this hypothesis would strengthen the conclusion that it is $R_{e}=\delta_{e}+\delta_{r}$ rather than $\delta_{r}$ or $\delta_{e}$ separately that is responsible for the relatively large contribution to $\bar{\lambda}$ and $d \bar{P} / d z$.

\section{Time oscillations of $d P(t) / d z$.}

The slow oscillations in time exhibited by the instantaneous linear power deposition $d P(t) / d z$ (Fig. 5), remain to be understood. They set in once $\lambda_{e}(t)$ reaches saturation, and their amplitude is much larger than the noise level in the simulation. It is puzzling that their period, $\sim 0.5 \mu \mathrm{s}$, is much longer than any typical time scale of the electrons. The evidence suggests that the oscillations are not due to numerical effects: their period and amplitude remained essentially unchanged when we doubled $M_{e}$, halved $\Delta t$, doubled $n_{e}^{\prime}$, or suppressed $\delta_{r}$. Since, in our simulations, the beam is not dynamical, the oscillations cannot be due to a coupling of the EC with the beam motion. We conjecture that they are triggered by subtle fluctuations of the coldest part of the EC phase space, namely, very low-energy electrons very close to the midplane of the chamber. Since these electrons do not mix very much with the rest of the EC phase space under the action of the beam passage, they have the potential for giving rise to longterm memory effects. A simple way to test this conjecture would be to heat up this portion of the EC phase space by adding small random bunch-to-bunch fluctuations in the bunch centroid - if the conjecture were correct, the oscillations would disappear.

$$
\text { D. } d \overline{\boldsymbol{P}} / d z \propto \overline{\boldsymbol{\lambda}}_{\boldsymbol{e}}
$$

The constancy of $D$ [Eq. (6)] for fixed $N_{b}$ and $t_{b}$ as the SEY model is varied is easier to understand, and better supported by our results, than the more general scaling represented by Eq. (7). Scaling formulas better than (7) might be easy to find and justify. These may have a practical usefulness to quickly estimate $d \bar{P} / d z$, given that $\bar{\lambda}_{e}$ is usually comparable to the neutralization density, $\bar{\lambda}_{b}=e N_{b} / s_{b}$. However, it should be kept in mind that the value of $K$ is probably sensitive to other quantities such as vacuum chamber shape and size, bunch length, and magnetic field configuration. It would be useful, therefore, to carry out the calculation of $K$ for other conditions, especially for field-free regions and quadrupole magnets.

\section{CONCLUSIONS}

For the LHC conditions $t_{b}=25 \mathrm{~ns}$ and $N_{b}=1 \times 10^{11}$, our main conclusions are: (a) The available cooling ca- 
pacity of the cryogenic system, which we take to be $\sim 1.7 \mathrm{~W} / \mathrm{m}$, will be exceeded by the power deposited by the EC if $\delta_{\max }$ exceeds $\sim 1.3$. (b) The EC power deposited will be comfortably below the cooling limit if $\delta_{\max } \leq 1.2$. (c) The EC power deposition is not strongly sensitive to the photoelectric yield. (d) The EC power deposition is sensitive to the amount of rediffused electrons in the SEY spectrum: if we neglect the rediffused electrons, we find good agreement with CERN simulation results [46]; if we include them at a level indicated by laboratory measurements of sample materials, our estimates for $d \bar{P} / d z$ are approximately doubled relative to the no-rediffused case. (e) We have described in fair detail the mechanism responsible for the relatively large contribution of the rediffused electrons.

For a bunch spacing $t_{b}=75 \mathrm{~ns}$ the EC power deposition exceeds the available cooling capacity of the cryogenic system only when $\delta_{\max }>2$ and $N_{b}>1.5 \times 10^{11}$ taken in combination.

The above conclusions for $d \bar{P} / d z$ are based on averages over a single batch injected into an empty chamber. These single-batch results underestimate $d \bar{P} / d z$ by $\sim 40 \%$ relative to the steady-state value, which is achieved after two or more batches. The actual LHC beam will have many gaps of various lengths, hence we can only conclude from our results that the actual power deposition for any given batch for nominal LHC conditions is in the range $2-2.8 \mathrm{~W} / \mathrm{m}$ or, equivalently, that the energy deposited is in the range $4-5.6 \mu \mathrm{J} / \mathrm{m}$ per batch, the actual value depending on which specific batch one considers: a batch immediately after a very long gap would deposit $\sim 4 \mu \mathrm{J} / \mathrm{m}$ of energy, while a batch following another batch would deposit $\sim 5.6 \mu \mathrm{J} / \mathrm{m}$. An accurate calculation of $d \bar{P} / d z$, therefore, needs to take into account the entire train structure of the beam. However, even if there were no long gaps in the whole beam, the available cooling capacity would not be exceeded if $\delta_{\max } \leq 1.2$.

The rediffused component of the secondary emission spectrum is important. It is sometimes assumed, incorrectly, that this component affects only the secondary electrons emitted at very low incident energy $E_{0}$. In fact, the backscattered component of the SEY, $R\left(E_{0}\right)$, while maximum at low $E_{0}$, does not decrease below $\sim 0.2-0.3$ even in the multi-keV range [71-73] for most materials. For the SEY model used here, $R\left(E_{0}\right) \sim 0.1-0.2$ in the energy range of interest, $E_{0} \sim 100-200 \mathrm{eV}$ (Fig. 2). The essential fact about the rediffused electrons is that they are emitted with a broad energy spectrum, $0 \leqq E \lesssim E_{0}$, hence their energies are typically higher than for true secondaries. The nominal parameters for $N_{b}$ and $t_{b}$, combined with chamber transverse dimensions of $\sim 4 \mathrm{~cm}$, place the LHC in a regime in which a fraction of only $\sim(10-20) \%$ of rediffused electrons roughly doubles $d \bar{P} / d z$ relative to the simplified case in which the rediffused electrons are neglected.
The ECE is a self-conditioning effect in the sense that, during normal machine operation, $\delta_{\max }$ gradually decreases owing to bombardment by the very same electrons that give rise to the ECE, as experienced has showed at the CERN Super Proton Synchrotron (SPS) [62] and the PSR [74]. It is generally expected that, as LHC operation progresses, so too will $\delta_{\max }$ eventually fall below a level where the EC will no longer be an operational limitation. It is therefore interesting to calculate how long it will take or, more precisely, how much integrated beam current will be required, for $\delta_{\max }$ to fall below $\sim 1.3$. Laboratory measurements at room temperature $[52,61]$ and at cryogenic temperatures $[55,57,63]$ show that the bombardment dose required for this level of conditioning to be reached is in the range $\sim 0.1-1 \mathrm{C} / \mathrm{cm}^{2}$. This dose can be translated into integrated beam current via simulations, as it has been done for the COLDEX experiment at the SPS $[55,57]$.

The importance of $\delta_{r}\left(E_{0}\right)$ raises an interesting question for the conditioning process: does the backscattered component decrease at the same rate that $\delta_{\max }$ does? Lab measurements [63] and indirect observations [74] strongly suggest that the backscattered component $R_{e}=\delta_{e}+\delta_{r}$ does not condition, while only the true secondary emission component gradually decreases with electron bombardment. Confirmation of these observations by actual experience at the LHC might imply a longer conditioning time than presently estimated. We intend to carry out a more complete analysis taking into account this evidence [75].

\section{ACKNOWLEDGMENTS}

We are indebted to F. Zimmermann and D. Schulte for discussions on their recent work on the ECE, and especially to F. Zimmermann, W. Fischer, V. Baglin, R. Cimino and A. Wolski for valuable comments on an earlier draft. We are grateful to NERSC for supercomputer support, and to the DOE Office of Science Undergraduate Laboratory Internship (SULI) Program for a summer internship for one of us (V.H. C.) at LBNL during the summer of 2005. This work was supported by the U.S. DOE under Contract No. DE-AC02-05CH11231 and by the US-LHC Accelerator Research Project (LARP).

[1] M. Izawa, Y. Sato, and T. Toyomasu, Phys. Rev. Lett. 74, 5044 (1995).

[2] K. Ohmi, Phys. Rev. Lett. 75, 1526 (1995).

[3] K. Ohmi, in Proceedings of the International Workshop on Collective Effects and Impedance for $B$ Factories: CEIBA95, edited by Y.H. Chin (KEK, Tsukuba, Japan, 1995), p. 288.

[4] S. Heifets, in Proceedings of the International Workshop on Collective Effects and Impedance for B Factories: CEIBA95 (Ref. [3]), p. 295.

[5] M. A. Furman and G. R. Lambertson, in Proceedings of the 5th European Particle Accelerator Conference, Sitges, Spain, 1996 (IOP, Bristol, 1996), p. 1087. 
[6] M. A. Furman and G. R. Lambertson, in Proceedings of the International Workshop on Multibunch Instabilities in Future Electron and Positron Accelerators MBI97, edited by Y.H. Chin (KEK, Tsukuba, Japan, 1997), p. 170.

[7] Z. Y. Guo, H. Huang, S. P. Li, D. K. Liu, L. Ma, Q. Qin, L. F. Wang, J. Q. Wang, S. H. Wang, C. Zhang, F. Zhou, Y.H. Chin, H. Fukuma, S. Hiramatsu, M. Izawa, T. Kasuga, E. Kikutabi, Y. Kobayashi, S. Kurokawa, K. Ohmi, Y. Sato, Y. Suetsugu, M. Tobiyama, K. Yokoya, and X.L. Zhang, in Proceedings of the International Workshop on Multibunch Instabilities in Future Electron and Positron Accelerators MBI97 (Ref. [6]), p. 150.

[8] F. Zimmermann, Report No. SLAC-PUB-7425/LHC Project Report 95, 1997.

[9] F. Zimmermann, in Proceedings of the International Workshop on Multibunch Instabilities in Future Electron and Positron Accelerators MBI97 (Ref. [6]), p. 221.

[10] O. Gröbner, Vacuum 47, 591 (1996).

[11] O. Gröbner, in Proceedings of the 10th International Conference on High Energy Accelerators, Serpukhov, 1977 (USSR Academy of Science, Moscow, 1977), pp. 277-282.

[12] O. Gröbner, in Proceedings of the 1997 Particle Accelerator Conference, Vancouver, BC, Canada, 1997 (IEEE, Piscataway, NJ, 1997), p. 3589.

[13] Proceedings of the International Workshop on Collective Effects and Impedance for B Factories: CEIBA95 (Ref. [3]).

[14] Proceedings of the Santa Fe Workshop on Electron Effects, edited by T. F. Wang and A. Jason (Los Alamos National Laboratory Report No. LA-UR-98-1601, 1998).

[15] Proceedings of the International Workshop on Multibunch Instabilities in Future Electron and Positron Accelerators MBI97 (Ref. [6]), KEK Proceedings 97-17, 1997.

[16] Workshop in Instabilities of High Intensity Hadron Beams in Rings, edited by T. Roser and S. Y. Zhang, AIP Conf. Proc. No. 496 (AIP, New York, 1999).

[17] Proceedings of the 8th Advanced Beam Dynamics ICFA Mini-Workshop on Two-Stream Instabilities in Particle Accelerators and Storage Rings, Santa Fe, NM, 2000, edited by K. Harkay and R. J. Macek, http://www.aps.anl.gov//News/Conferences/2000/icfa/two-stream.html

[18] Proceedings of the International Workshop on Two-Stream Instabilities in Particle Accelerator and Storage Rings, 2001, KEK, Tsukuba-shi, Japan, http://conference.kek.jp/ two-stream/

[19] Proceedings of the 20th ICFA Advanced Beam Dynamics Workshop on High Intensity High Brightness Hadron Beams HB2002, FNAL, 2002, edited by W. Chou and Y. Mori, http://proceedings.aip.org/proceedings/confproceed/642.jsp

[20] Proceedings of the Mini-Workshop on Electron-Cloud Simulations for Proton and Positron Beams ECLOUD'02, CERN, 2002, edited by F. Zimmermann and G. Rumolo, CERN Yellow Report No. CERN-2002001; http://slap.cern.ch/collective/ecloud02/

[21] Proceedings of the 13th ICFA Beam Dynamics MiniWorkshop on Beam-Induced Pressure Rise in Rings, $B N L, 2003$, http://www.c-ad.bnl.gov/icfa/

[22] Proceedings of the 31st ICFA Advanced Beam Dynamics Workshop on Electron-Cloud Effects ECLOUD'04, Napa,
$C A, 2004$, edited by M. Furman, S. Henderson, and F. Zimmermann, CERN Yellow Report No. CERN2005-001/CARE-Conf-05-001-HHH/LBNL-56372/SNS10400000-TR0024-R00; http://icfa-ecloud04.web.cern.ch / icfa-ecloud 04/

[23] Proceedings of the 33rd ICFA Advanced Beam Dynamics Workshop on High Intensity and High Brightness Hadron Beams, ICFA-HB2004, Bensheim, Germany, 2004, edited by I. Hofmann and J.-M. Lagniel, http:// www.gsi.de / search/events/conferences/ICFA-HB2004 / index_e.html

[24] Proceedings of the 1st CARE-HHH-APD Workshop on Beam Dynamics in Future Hadron Colliders and Rapidly Cycling High-Intensity Synchrotrons, HHH2004, CERN, 2004, edited by F. Ruggiero and F. Zimmermann, http://care-hhh.web.cern.ch/care-hhh/HHH-2004/

[25] ICFA Beam Dynamics Newsletter No. 33, http://wwwbd.fnal.gov/icfabd/

[26] F. Zimmermann, ICFA Beam Dynamics Newsletter No. 31, http://www-bd.fnal.gov/icfabd/

[27] F. Zimmermann, Phys. Rev. ST Accel. Beams 7, 124801 (2004).

[28] K. Harkay, in Proceedings of the 31st ICFA Advanced Beam Dynamics Workshop on Electron-Cloud Effects ECLOUD'04, Napa, CA, 2004 (Ref. [22]), p. 9.

[29] V. Dudnikov, in Proceedings of the Particle Accelerator Conference, Chicago, IL, 2001 (IEEE, Piscataway, NJ, 2001); Report No. TPPH094.

[30] A review of the early work, with numerous references, has been given by V. Dudnikov, in Proceedings of the 13th ICFA Beam Dynamics Mini-Workshop on Beam-Induced Pressure Rise in Rings, BNL, 2003 (Ref. [21]).

[31] D. Neuffer, E. Colton, D Fitzgerald, T. Hardek, R. Huston, R. Macek, M. Plum, H. Thiessen, and T.-S. Wang, Nucl. Instrum. Methods Phys. Res., Sect. A 321, 1 (1992).

[32] M.A. Furman, No. LBNL-41482/CBP Note 247/LHC Project Report 180, 1998.

[33] G. Rumolo, F. Ruggiero, and F. Zimmermann, Phys. Rev. ST Accel. Beams 4, 012801 (2001).

[34] Electron-Cloud in the LHC Web site, http://ab-abprlc.web.cern.ch/ab\%2Dabp\%2Drlc\%2Decloud/

[35] O. S. Brüning, LHC Project Note No. 102, 1997.

[36] M.A. Furman, in Proceedings of the International Workshop on Multibunch Instabilities in Future Electron and Positron Accelerators MBI97 (Ref. [15]), p. 234.

[37] J. S. Berg, LHC Project Note No. 97, 1997.

[38] G. V. Stupakov, LHC Project Report No. 141, 1997.

[39] F. Zimmermann, in LHC MAC Meeting No. 17, 2005, http: //mgt-lhc-machine-advisory-committee.web.cern.ch/ mgt-lhc-machine-advisory-committee / lhcmac17 / Closed Session/Zimmermann.pdf

[40] M. A. Furman and M. Pivi, in Proceedings of the Particle Accelerator Conference, Chicago, IL, 2001 (Ref. [32]), p. 1898.

[41] F. Zimmermann, in Proceedings of the Mini-Workshop on Electron-Cloud Simulations for Proton and Positron Beams ECLOUD'02, CERN, 2002 (Ref. [20]), p. 47.

[42] M. Furman and M. Pivi, in Proceedings of the MiniWorkshop on Electron-Cloud Simulations for Proton and Positron Beams ECLOUD'02, CERN, 2002 (Ref. [20]). This talk is not in the proceedings, but can 
be accessed from the talks Web site, http://cern.ch/confecloud02/talks/furman_ECLOUD02_3_02.pdf

[43] M. A. Furman and M. Pivi, in Proceedings the 8th European Particle Accelerator Conference, Paris, 2002 (EPS-IGA and CERN, Geneva, 2002); Report No. WEPDO005.

[44] M. A. Furman, in Proceedings of the Particle Accelerator Conference, Portland, OR, 2003 (IEEE, Piscataway, NJ, 2003); Report No. TOPC001.

[45] LHC Design Report No. CERN-2004-003, 2004.

[46] F. Zimmermann, in LTC Meeting No. 40, CERN, 2005, http: // edms.cern.ch / lhc_proj / plsql / lhcp.p.?p_number= 7700

[47] V. Baglin, I. R. Collins, and O. Gröbner, in Proceedings the European Particle Accelerator Conference, Stockholm, 1998 (IOP, London, 1998); Report No. TUP18H.

[48] V. Baglin, O. Brüning, R. Calder, F. Caspers, I. R. Collins, O. Gröbner, N. Hilleret, J.-M. Laurent, M. Morvillo, M. Pivi, and F. Ruggiero, in Proceedings the European Particle Accelerator Conference, Stockholm, 1998 (Ref. [47]); Report No. MOP10C.

[49] V.V. Anashin, O.B. Malyshev, N. V. Fedorov, V.P. Nazmov, B. G. Goldenberg, I. R. Collins, and O. Gröbner, Nucl. Instrum. Methods Phys. Res., Sect. A 448, 76 (2000).

[50] V. Baglin, in Proceedings of the CERN E-Cloud Meeting, 2000, http://wwwslap.cern.ch/collective/electron-cloud/ baglin/EPA_sey-14-04-2000.pdf

[51] V. Baglin, J. Bojko, O. Gröbner, B. Henrist, N. Hilleret, C. Scheuerlein, and M. Taborelli, in Proceedings the European Particle Accelerator Conference, Vienna, 2000 (EPS, Geneva, 2000); Report No. THXF102.

[52] V. Baglin, I. Collins, B. Henrist, N. Hilleret, and G. Vorlaufer, LHC Project Report No. 472, 2001.

[53] V. Baglin, I. Collins, O. Gröbner, B. Henrist, N. Hilleret, G. Vorlaufer, in Proceedings of the International Workshop on Two-Stream Instabilities in Particle Accelerator and Storage Rings, 2001, KEK, Tsukuba-shi, Japan (Ref. [18]).

[54] B. Henrist, N. Hilleret, M. Jiménez, C. Scheuerlein, M. Taborelli, and G. Vorlaufer, Proceedings of the MiniWorkshop on Electron-Cloud Simulations for Proton and Positron Beams ECLOUD'02, CERN, 2002 (Ref. [20]), p. 75.

[55] V. Baglin and B. Jenninger, Report No. LHC-PR-721, 2004; in Proceedings of the 31st ICFA Advanced Beam Dynamics Workshop on Electron-Cloud Effects ECLOUD'04, Napa, CA, 2004 (Ref. [22]), p. 123.

[56] N. Mahne, V. Baglin, I. R. Collins, A. Giglia, L. Pasquali, M. Pedio, S. Nannarone, and R. Cimino, Appl. Surf. Sci. 235, 221 (2004).

[57] V. Baglin and B. Jenninger, in APC Meeting No. 47, 2005; http://ab-div.web.cern.ch/ab-div/Meetings/APC/

[58] V. Baglin, CERN Web site on Secondary Electron Literature, http://at-div-vac.web.cern.ch/at-div-vac/VACPAGES/ ps/Phys\&tech/Phys/Ecloud/SEY/SEY_paper.html
[59] Reference [45], Sec. 12.3.1.

[60] D. Schulte and F. Zimmermann, in Proceedings of the 31st ICFA Advanced Beam Dynamics Workshop on ElectronCloud Effects ECLOUD'04, Napa, CA, 2004 (Ref. [22]), p. 143.

[61] R. E. Kirby and F. K. King, Nucl. Instrum. Methods Phys. Res., Sect. A 469, 1 (2001).

[62] J. M. Jiménez, G. Arduini, P. Collier, G. Ferioli, B. Henrist, N. Hilleret, L. Jensen, K. Weiss, and F. Zimmermann, LHC Project Report No. 632, 2003.

[63] R. Cimino and I.R. Collins, LHC Project Report No. 669, 2003; in Proceedings 8th European Vacuum Conference and 2nd Annual Conference of the German Vacuum Society, Berlin, Germany, 2003, edited by J.-J. Pireaux, R. Wiesendanger, and K. Jousten (Elsevier, Amsterdam, 2004); Appl. Surf. Sci. 235, 231 (2004).

[64] M. A. Furman and M. T.F. Pivi, Phys. Rev. ST Accel. Beams 5, 124404 (2002).

[65] M. A. Furman and M. T. F. Pivi, Report No. LBNL-52807/ SLAC-PUB-9912, 2003.

[66] H. Bruining, Physics and Applications of Secondary Electron Emission (Pergamon Press, McGraw-Hill Book Co., New York, 1954).

[67] M. A. Furman, M. Pivi, K.C. Harkay, and R. A. Rosenberg, in Proceedings of the Particle Accelerator Conference, Chicago, IL, 2001 (Ref. [29]), p. 679.

[68] M. A. Furman and M. Pivi, in Proceedings of the Particle Accelerator Conference, Chicago, IL, 2001 (Ref. [29]), p. 707.

[69] G. Rumolo and F. Zimmermann, in Proceedings of the Mini-Workshop on Electron-Cloud Simulations for Proton and Positron Beams ECLOUD'02, CERN, 2002 (Ref. [20]), p. 97.

[70] R. Cimino, I. R. Collins, M. A. Furman, M. Pivi, F. Ruggiero, G. Rumolo, and F. Zimmermann, Phys. Rev. Lett. 93, 014801 (2004).

[71] D. C. Joy, Scanning 17, 270 (1995). The database can be downloaded from http://pciserver.bio.utk.edu/metrology/ $\mathrm{htm} / \mathrm{home}$.shtml

[72] Backscattered electron data and simulation tools can be obtained from the Web site "MC-SET: Monte Carlo Simulation of Electron Trajectories," http://www.napchan.com/

[73] M. Dapor, Electron-Beam Interactions With Solids: Application of the Monte Carlo Method to Electron Scattering Problems, Springer Tracts in Modern Physics Vol. 186 (Springer-Verlag, New York, 2003).

[74] R. J. Macek, A. A. Browman, M.J. Borden, D. H. Fitzgerald, R.C. McCrady, T. Spickermann, and T.J. Zaugg, in Proceedings of the 31st ICFA Advanced Beam Dynamics Workshop on ElectronCloud Effects ECLOUD'04, Napa, CA, 2004 (Ref. [22]), p. 63.

[75] M. A. Furman (to be published). 